\title{
Carleson measures for spaces of Dirichlet type
}

\author{
Daniel Girela and José Ángel Peláez
}

Dedicated to Albert Baernstein on the occasion of his 65th birthday

\begin{abstract}
If $0<p<\infty$ and $\alpha>-1$, the space $\mathcal{D}_{\alpha}^{p}$ consists of those functions $f$ which are analytic in the unit disc $\mathbb{D}$ and have the property that $f^{\prime}$ belongs to the weighted Bergman space $A_{\alpha}^{p}$. In 1999, Z. Wu obtained a characterization of the Carleson measures for the spaces $\mathcal{D}_{\alpha}^{p}$ for certain values of $p$ and $\alpha$. In particular, he proved that, for $0<p \leq 2$, the Carleson measures for the space $\mathcal{D}_{p-1}^{p}$ are precisely the classical Carleson measures. Wu also conjectured that this result remains true for $2<p<\infty$. In this paper we prove that this conjecture is false. Indeed, we prove that if $2<p<\infty$, then there exists $g$ analytic in $\mathbb{D}$ such that the measure $\mu_{g, p}$ on $\mathbb{D}$ defined by $d \mu_{g, p}(z)=$ $\left(1-|z|^{2}\right)^{p-1}\left|g^{\prime}(z)\right|^{p} d x d y$ is not a Carleson measure for $\mathcal{D}_{p-1}^{p}$ but is a classical Carleson measure. We obtain also some sufficient conditions for multipliers of the spaces $\mathcal{D}_{p-1}^{p}$.
\end{abstract}

Mathematics Subject Classification (2000). Primary 30H05; Secondary 46J15.

Keywords. Carleson measures, Bergman spaces, Dirichlet spaces, Multipliers.

\section{Introduction}

We denote by $\mathbb{D}$ the unit $\operatorname{disc}\{z \in \mathbb{C}:|z|<1\}$ and by $\mathcal{H o l}(\mathbb{D})$ the space of all analytic functions in $\mathbb{D}$. Also, $H^{p}(0<p \leq \infty)$ are the classical Hardy spaces of analytic functions in $\mathbb{D}$ (see [6] and [10]).

If $E$ is a measurable subset of the unit circle $\mathbb{T}=\partial \mathbb{D}$, we write $|E|$ for the Lebesgue measure of $E$. If $I \subset \mathbb{T}$ is an interval, the Carleson square $S(I)$ is defined as

$$
S(I)=\left\{r e^{i t}: e^{i t} \in I, \quad 1-\frac{|I|}{2 \pi} \leq r<1\right\} .
$$

The authors are partially supported by grants from "El Ministerio de Educación y Ciencia, Spain" and FEDER (MTM2004-00078 and MTM2004-21420-E) and by a grant from "La Junta de Andalucía" (FQM-210). 
Carleson [5] (see also Theorem 9.3 of [6]) proved that if $0<p<\infty$ and $\mu$ is a positive Borel measure in $\mathbb{D}$ then $H^{p} \subset L^{p}(d \mu)$ if and only if there exists a positive constant $C$ such that

$$
\mu(S(I)) \leq C|I| \text {, for every interval } I \subset \mathbb{T} .
$$

The measures $\mu$ which satisfy this condition will be called classical Carleson measures.

If $0<p<\infty$ and $\alpha>-1$, the weighted Bergman space $A_{\alpha}^{p}$ consists of those $f \in \mathcal{H o l}(\mathbb{D})$ such that

$$
\|f\|_{A_{\alpha}^{p}} \stackrel{\text { def }}{=}\left((\alpha+1) \int_{\mathbb{D}}(1-|z|)^{\alpha}|f(z)|^{p} d A(z)\right)^{1 / p}<\infty .
$$

The unweighted Bergman space $A_{0}^{p}$ is simply denoted by $A^{p}$. Here, $d A(z)=\frac{1}{\pi} d x d y$ denotes the normalized Lebesgue area measure in $\mathbb{D}$. We refer to [7] and [15] for the theory of these spaces.

The space $\mathcal{D}_{\alpha}^{p}(0<p<\infty, \alpha>-1)$ consists of those $f \in \mathcal{H}$ ol $(\mathbb{D})$ such that $f^{\prime} \in A_{\alpha}^{p}$. Hence, if $f$ is analytic in $\mathbb{D}$, then $f \in \mathcal{D}_{\alpha}^{p}$ if and only if

$$
\|f\|_{\mathcal{D}_{\alpha}^{p}}^{p} \stackrel{\text { def }}{=}|f(0)|^{p}+\left\|f^{\prime}\right\|_{A_{\alpha}^{p}}^{p}<\infty .
$$

If $p<\alpha+1$ then it is well known that $\mathcal{D}_{\alpha}^{p}=A_{\alpha-p}^{p}$ (see, e. g. Theorem 6 of [9]). It is trivial that $D_{1}^{2}=H^{2}$. The spaces $\mathcal{D}_{\alpha}^{p}$ are called Dirichlet spaces if $p \geq \alpha+1$. In particular, the space $\mathcal{D}_{0}^{2}$ is the classical Dirichlet space.

A positive Borel measure $\mu$ in $\mathbb{D}$ is said to be a Carleson measure for $A_{\alpha}^{p}$ (respectively, a Carleson measure for $\mathcal{D}_{\alpha}^{p}$ ) if $A_{\alpha}^{p} \subset L^{p}(d \mu)$ (respectively, $\mathcal{D}_{\alpha}^{p} \subset$ $\left.L^{p}(d \mu)\right)$.

The Carleson measures for $A_{\alpha}^{p}$ are characterized in the following theorem.

Theorem A. Suppose that $0<p<\infty$ and $\alpha>-1$, and let $\mu$ be a positive Borel measure on $\mathbb{D}$. Then $\mu$ is a Carleson measure for $A_{\alpha}^{p}$ if and only if there exists a positive constant $C$ such that $\mu(S(I)) \leq C|I|^{\alpha+2}$, for every interval $I \subset \mathbb{T}$.

Theorem A was obtained by Oleinik and Pavlov [19, 20] (see also the works of Stegenga [21] and Hastings [14] where the result is proved for certain values of $p$ and $\alpha$ ). Luecking $[16,17]$ (see also Section 2.10 of [7]) obtained another characterizazion of the Carleson measures for $A_{\alpha}^{p}$ which involves the pseudohyperbolic metric.

Z. Wu [23] and Arcozzi, Rochberg and Sawyer [1] obtained a characterization of the Carleson measures for the spaces $\mathcal{D}_{\alpha}^{p}$ for certain values of $p, \alpha$. In particular, parts (c) and (d) of Theorem 1 of [23] (see also Theorem 2.1 of [22]), yield the following result.

Theorem B. Suppose that $0<p \leq 2$ and let $\mu$ be a positive Borel measure on $\mathbb{D}$, then $\mu$ is a Carleson measure for $\mathcal{D}_{p-1}^{p}$ if and only if $\mu$ is a classical Carleson measure. 


\section{The main result}

Wu conjectured in p. 149 of [23] that the conclusion of Theorem B is also true for $2<p<\infty$. In this paper we shall see that this conjecture is not true. Indeed, we shall prove the following result.

Theorem 2.1. Suppose that $2<p<\infty$. Then there exists a function $g \in \mathcal{H}$ ol $(\mathbb{D})$ such that the measure $\mu_{g, p}$ on $\mathbb{D}$ given by $d \mu_{g, p}(z)=\left(1-|z|^{2}\right)^{p-1}\left|g^{\prime}(z)\right|^{p} d A(z)$ is not a Carleson measure for $\mathcal{D}_{p-1}^{p}$ but is a classical Carleson measure.

Note that if $\mu$ is a Carleson measure for $\mathcal{D}_{p-1}^{p}$ then it is a classical Carleson measure (see [23, Lemma 3. 1]). Theorem 2.1 shows that $\mu$ being a classical Carleson measure is not enough to deduce that $\mu$ is a Carleson measure for $\mathcal{D}_{p-1}^{p}$ $(2<p<\infty)$. However, it is easy to prove the following result.

Proposition 2.2. Suppose that $2<p<\infty$ and let $\mu$ be a positive Borel measure on $\mathbb{D}$. If there exist $C>0$ and $\varepsilon>0$ such that

$$
\mu(S(I)) \leq C|I|^{1+\varepsilon},
$$

for all intervals $I \subset \mathbb{T}$, then $\mu$ is a Carleson measure for $\mathcal{D}_{p-1}^{p}$.

Theorem 2.1 and Proposition 2.2 will be proved in Section 4 . Section 3 will be devoted to obtain several results that will be needed in the proof of Theorem 2.1 and which may be of independent interest. In particular, Theorem 3.1 and Theorem 3.2 will be used in Section 5 to obtain sufficient conditions for multipliers of the spaces $\mathcal{D}_{p-1}^{p}, 0<p<2$.

As usual, throughout this paper the letter $C$ denotes a positive constant that may change from one step to the next.

\section{Preliminary results}

We start obtaining a condition on the Taylor coefficients of a function $g \in \mathcal{H}$ ol $(\mathbb{D})$ which implies that the measure $\mu_{g, p}$ on $\mathbb{D}$ defined as in Theorem 2.1 is a classical Carleson measure.

Theorem 3.1. Let $g$ be an analytic function in $\mathbb{D}, g(z)=\sum_{n=0}^{\infty} a_{n} z^{n}(z \in \mathbb{D})$. If $0<p<\infty$ and

$$
\sum_{n=0}^{\infty}\left(\sum_{k \in I(n)}\left|a_{k}\right|\right)^{p}<\infty
$$

then the measure $\mu_{g, p}$ on $\mathbb{D}$ defined by $d \mu_{g, p}=\left(1-|z|^{2}\right)^{p-1}\left|g^{\prime}(z)\right|^{p} d A(z)$ is a classical Carleson measure.

Here and all over the paper, for $n=0,1, \ldots$, we let $I(n)$ be the set of the integers $k$ such that $2^{n} \leq k<2^{n+1}$.

Theorem 3.1 improves part (i) of Theorem 1 of [12] which asserts that (3.1) implies that $g \in \mathcal{D}_{p-1}^{p}$. 
Proof of Theorem 3.1. Using Lemma 3.3 in p. 239 of [10], we see that it suffices to prove that

$$
\sup _{a \in \mathbb{D}} \int_{\mathbb{D}} \frac{\left(1-|a|^{2}\right)\left(1-|z|^{2}\right)^{p-1}}{|1-\bar{a} z|^{2}}\left|g^{\prime}(z)\right|^{p} d A(z)<\infty .
$$

Now, using Theorem 1 of [18], we deduce that there is a constant $C_{p}$ which depends only on $p$ such that, for every $a \in \mathbb{D}$,

$$
\begin{aligned}
& \int_{\mathbb{D}} \frac{\left(1-|a|^{2}\right)\left(1-|z|^{2}\right)^{p-1}}{|1-\bar{a} z|^{2}}\left|g^{\prime}(z)\right|^{p} d A(z) \\
& \leq C_{p} \int_{0}^{1}\left(1-|a|^{2}\right)\left(1-r^{2}\right)^{p-1}\left(\sum_{n=1}^{\infty} n\left|a_{n}\right| r^{n-1}\right)^{p}\left(\int_{0}^{2 \pi} \frac{1}{\left|1-\bar{a} r e^{i t}\right|^{2}} d t\right) d r \\
& \leq C_{p} \int_{0}^{1} \frac{\left(1-|a|^{2}\right)\left(1-r^{2}\right)^{p-1}}{1-|a|^{2} r^{2}}\left(\sum_{n=1}^{\infty} n\left|a_{n}\right| r^{n-1}\right)^{p} d r \\
& \leq C_{p} \int_{0}^{1}\left(1-r^{2}\right)^{p-1}\left(\sum_{n=1}^{\infty} n\left|a_{n}\right| r^{n-1}\right)^{p} d r \\
& \leq C_{p} \sum_{n=0}^{\infty} 2^{-n p}\left(\sum_{k \in I(n)} k\left|a_{k}\right|\right)^{p} \\
& \leq C_{p} \sum_{n=0}^{\infty}\left(\sum_{k \in I(n)}\left|a_{k}\right|\right)^{p}=A_{p}<\infty .
\end{aligned}
$$

Hence, we have proved (3.2). This finishes the proof.

Using Proposition 2.1 of [4] (see also Proposition A of [12]), we obtain that if $g \in \mathcal{H} o l(\mathbb{D})$ is given by a power series with Hadamard gaps,

$$
g(z)=\sum_{k=1}^{\infty} a_{k} z^{n_{k}}(z \in \mathbb{D}), \text { with } n_{k+1} \geq \lambda n_{k} \text { for all } k, \text { for some } \lambda>1,
$$

then, for every $p \in(0, \infty)$,

$$
g \in \mathcal{D}_{p-1}^{p} \Longleftrightarrow \sum_{k=1}^{\infty}\left|a_{k}\right|^{p}<\infty .
$$

Our next theorem is an improvement of this result.

Theorem 3.2. Suppose that $0<p<\infty$ and let $g$ be an analytic function in $\mathbb{D}$ which is given by a power series with Hadamard gaps,

$$
g(z)=\sum_{k=1}^{\infty} a_{k} z^{n_{k}} \quad(z \in \mathbb{D}) \quad \text { with } n_{k+1} \geq \lambda n_{k}, \text { for all } k \quad(\lambda>1),
$$

then, the following conditions are equivalent: 
(a) The measure $\mu_{g, p}$ on $\mathbb{D}$ defined by $d \mu_{g, p}=\left(1-|z|^{2}\right)^{p-1}\left|g^{\prime}(z)\right|^{p} d A(z)$ is a classical Carleson measure.

(b) $g \in \mathcal{D}_{p-1}^{p}$.

(c) $\sum_{k=1}^{\infty}\left|a_{k}\right|^{p}<\infty$.

Proof. We already know that $(b) \Leftrightarrow(c)$. Trivially, (a) implies that $\mu_{g, p}$ is a finite measure and, hence, $g \in \mathcal{D}_{p-1}^{p}$. Thus, we have seen that $(a) \Rightarrow(b)$. Consequently, it only remains to prove that $(c) \Rightarrow(a)$.

So take $g \in \mathcal{H}$ ol $(\mathbb{D})$ which is given by a power series with Hadamard gaps

$$
g(z)=\sum_{k=1}^{\infty} a_{k} z^{n_{k}}, \quad \text { with } \frac{n_{k+1}}{n_{k}} \geq \lambda>1, \text { for all } \mathrm{k},
$$

and suppose that $\sum_{k=1}^{\infty}\left|a_{k}\right|^{p}<\infty$. Using the gap condition, we see that there are at most $C_{\lambda}=\log _{\lambda} 2+1$ of the $n_{k}{ }^{\prime} s$ in the set $I(n)$. Then there exists a constant $C_{\lambda, p}>0$ such that

$$
\sum_{n=0}^{\infty}\left(\sum_{k \in I(n)}\left|a_{k}\right|\right)^{p} \leq C_{\lambda, p} \sum_{k=1}^{\infty}\left|a_{k}\right|^{p}<\infty .
$$

Using Theorem 3.1, we deduce that $\mu_{g, p}$ is a classical Carleson measure. Thus, we have proved that $(c) \Rightarrow(a)$, as needed. This finishes the proof.

We need to introduce some notation to state our last result in this section. If $f \in \mathcal{H} o l(\mathbb{D}), 0<p<\infty$ and $0 \leq r<1$, we set, as usual,

Notice that

$$
M_{p}(r, f)=\left(\frac{1}{2 \pi} \int_{-\pi}^{\pi}\left|f\left(r e^{i t}\right)\right|^{p} d t\right)^{1 / p}
$$

$$
g \in \mathcal{D}_{p-1}^{p} \Leftrightarrow \int_{0}^{1}(1-r)^{p-1} M_{p}^{p}\left(r, g^{\prime}\right) d r<\infty .
$$

It is well known (see Theorem 8.20 in p. 215 of Vol. I of [26]) that if $f \in \mathcal{H o l}(\mathbb{D})$ is given by a power series with Hadamard gaps and $0<p<\infty$, then $M_{2}(r, f) \asymp$ $M_{p}(r, f)$. It follows that if $g \in \mathcal{H}$ ol $(\mathbb{D})$ is given by a power series with Hadamard gaps then

$$
g \in \mathcal{D}_{p-1}^{p} \quad \Leftrightarrow \quad \int_{0}^{1}(1-r)^{p-1} M_{2}^{p}\left(r, g^{\prime}\right) d r<\infty .
$$

Our next theorem asserts that this result is sharp in a strong sense.

Theorem 3.3. Suppose that $0<p<\infty$ and let $\phi$ be a positive and increasing function defined in $(0,1)$ such that

$$
\int_{0}^{1}(1-r)^{p-1} \phi^{p}(r) d r<\infty .
$$

Then there exists a function $g \in \mathcal{D}_{p-1}^{p}$ given by a power series with Hadamard gaps such that

$$
M_{2}\left(r, g^{\prime}\right) \geq \phi(r) \quad \text { for all } r \in(0,1) \text {. }
$$


The proof of Theorem 3.3 is very similar to that of Theorem D of [11]. Proof of Theorem 3.3. Set $r_{k}=1-2^{-k}, k=1,2, \ldots$ Since $\phi$ is increasing

$$
\begin{aligned}
\int_{0}^{1}(1-r)^{p-1} \phi^{p}(r) d r & \geq \sum_{k=1}^{\infty} \int_{r_{k}}^{r_{k+1}}(1-r)^{p-1} \phi^{p}(r) d r \\
& \geq \sum_{k=1}^{\infty}\left(r_{k+1}-r_{k}\right)\left(1-r_{k+1}\right)^{p-1} \phi^{p}\left(r_{k}\right) \\
& =2^{-p} \sum_{k=1}^{\infty} 2^{-k p} \phi^{p}\left(r_{k}\right) .
\end{aligned}
$$

Hence,

$$
\sum_{k=1}^{\infty} 2^{-k p} \phi^{p}\left(r_{k}\right)<\infty
$$

Set

$$
g(z)=\phi\left(r_{1}\right) z+e^{4} \sum_{k=1}^{\infty} 2^{-k} \phi\left(r_{k}\right) z^{2^{k}}, \quad z \in \mathbb{D} .
$$

Then $g$ is an analytic function in $\mathbb{D}$ which is given by a power series with Hadamard gaps. Using Theorem 3.2 and (3.6), we deduce that $g \in \mathcal{D}_{p-1}^{p}$.

We have

$M_{2}^{2}\left(r, g^{\prime}\right)=\phi^{2}\left(r_{1}\right)+e^{8} \sum_{k=1}^{\infty} \phi^{2}\left(r_{k}\right) r^{2^{k+1}-2} \geq \phi^{2}\left(r_{1}\right)+e^{8} \sum_{k=1}^{\infty} \phi^{2}\left(r_{k}\right) r^{2^{k+1}}, 0<r<1$.

Since $\phi$ is increasing, we deduce that

$$
M_{2}^{2}\left(r, g^{\prime}\right) \geq \phi^{2}\left(r_{1}\right) \geq \phi^{2}(r), \quad 0<r \leq r_{1} .
$$

Now, using the elementary inequality $\left(1-n^{-1}\right)^{n} \geq e^{-2}(n \geq 2)$ and bearing in mind that $\phi$ is increasing, we see that, for $j \geq 1$ and $r_{j} \leq r \leq r_{j+1}$,

$$
M_{2}^{2}\left(r, g^{\prime}\right) \geq e^{8} \sum_{k=1}^{\infty} \phi^{2}\left(r_{k}\right) r^{2^{k+1}} \geq e^{8} \phi^{2}\left(r_{j+1}\right) r^{2^{j+2}} \geq e^{8} \phi^{2}(r)\left(1-2^{-j}\right)^{4 \cdot 2^{j}} \geq \phi^{2}(r) \text {. }
$$

This together with (3.7) implies that $M_{2}\left(r, g^{\prime}\right) \geq \phi(r)$, for all $r \in(0,1)$, and finishes the proof.

\section{Proof of the main results}

Proof of Proposition 2.2. Suppose $p, \mu, C$ and $\varepsilon$ are as in Proposition 2.2. Take $f \in \mathcal{D}_{p-1}^{p}$. Then it is easy to see that

$$
M_{p}\left(r, f^{\prime}\right)=\mathrm{o}\left(\frac{1}{1-r}\right), \quad \text { as } r \rightarrow 1
$$


Then it follows easily that $M_{p}(r, f)=\mathrm{O}\left(\log \frac{1}{1-r}\right)$, as $r \rightarrow 1$. Actually, Theorem 1 of [13] implies that

$$
M_{p}(r, f)=\mathrm{O}\left(\left(\log \frac{1}{1-r}\right)^{\beta}\right), \quad \text { as } r \rightarrow 1,
$$

for all $\beta>\frac{1}{2}$. Then it is clear that $f \in A_{\alpha}^{p}$, for every $\alpha>-1$. Consequently, we have proved that $\mathcal{D}_{p-1}^{p} \subset A_{\alpha}^{p}$, for every $\alpha>-1$. In particular, $\mathcal{D}_{p-1}^{p} \subset A_{-1+\varepsilon}^{p}$. Now, Theorem A implies that $\mu$ is a Carleson measure for $A_{-1+\varepsilon}^{p}$ and then it follows that $\mu$ is also a Carleson measure for $\mathcal{D}_{p-1}^{p}$.

Proof of Theorem 2.1. Suppose that $2<p<\infty$. Take two positive numbers $\alpha$ and $\varepsilon$ such that $\frac{1}{p}<\alpha<\frac{1}{2}$ and $0<\varepsilon<\frac{1}{2}-\alpha$ and define

$$
f(z)=\sum_{k=0}^{\infty} \frac{1}{k^{\frac{1}{p}+\varepsilon}} z^{2^{k}}, \quad z \in \mathbb{D} .
$$

Using Theorem 3.2 we see that $f \in \mathcal{D}_{p-1}^{p}$. Also, it is easy to see that there exist $r_{0} \in(0,1)$ and $C>0$ such that

$$
M_{2}(r, f) \geq C\left(\log \frac{1}{1-r}\right)^{\frac{1}{2}-\frac{1}{p}-\varepsilon} \quad r_{0} \leq r<1 .
$$

Since $f$ is given by a power series with Hadamard gaps, using Theorem 8.25 in chapter $V$ of Vol. I of [26], we see that there exist two absolute constants $A>0$ and $B>0$ such that for every $r \in(0,1)$ the set

$$
E_{r}=\left\{t \in[0,2 \pi]:\left|f\left(r e^{i t}\right)\right|>B M_{2}(r, f)\right\}
$$

has Lebesgue measure greater than or equal to $A$,

$$
\left|E_{r}\right| \geq A, \quad 0<r<1 \text {. }
$$

Define

$$
\phi(r)=\frac{1}{(1-r)\left[\log \left(\frac{e^{\alpha}}{1-r}\right)\right]^{\alpha}}, \quad 0 \leq r<1 .
$$

Then $\phi$ is an increasing function defined in $(0,1)$ and $\int_{0}^{1}(1-r)^{p-1} \phi^{p}(r) d r<\infty$. Using Theorem 3.3, we see that there exists a function $g \in \mathcal{D}_{p-1}^{p}$ which is given by a power series with Hadamard gaps and such that

$$
M_{2}\left(r, g^{\prime}\right) \geq \phi(r), \quad r \in(0,1) .
$$

Now, Theorem 3.2 implies that the measure $\mu_{g, p}$ is a classical Carleson measure. Using Hölder's inequality, Lemma 6.5 in Chapter V of Vol. I of [26] and (4.3), we deduce that there exists a positive constant $C_{1}$ such that

$$
\begin{aligned}
& \int_{E_{r}}\left|g^{\prime}\left(r e^{i t}\right)\right|^{p} d t \geq\left|E_{r}\right|^{1-\frac{p}{2}}\left(\int_{E_{r}}\left|g^{\prime}\left(r e^{i t}\right)\right|^{2} d t\right)^{p / 2} \\
\geq & C_{1}\left|E_{r}\right| M_{2}^{p}\left(r, g^{\prime}\right) \geq C_{1} A M_{2}^{p}\left(r, g^{\prime}\right), \quad 0<r<1 .
\end{aligned}
$$


Hence, setting $C=C_{1} A$, we have

$$
\int_{E_{r}}\left|g^{\prime}\left(r e^{i t}\right)\right|^{p} d t \geq C M_{2}^{p}\left(r, g^{\prime}\right), \quad 0<r<1 .
$$

Bearing in mind the definition of the sets $E_{r}(0<r<1)$ and using (4.6), (4.1), (4.5) and the fact that $\alpha<\frac{1}{2}-\varepsilon$, we obtain

$$
\begin{aligned}
& \int_{\mathbb{D}}\left(1-|z|^{2}\right)^{p-1}\left|g^{\prime}(z)\right|^{p}|f(z)|^{p} d A(z) \\
& \geq C \int_{r_{0}}^{1}(1-r)^{p-1} \int_{E_{r}}\left|g^{\prime}\left(r e^{i t}\right)\right|^{p}\left|f\left(r e^{i t}\right)\right|^{p} d t d r \\
& \geq C \int_{r_{0}}^{1}(1-r)^{p-1} M_{2}^{p}(r, f) \int_{E_{r}}\left|g^{\prime}\left(r e^{i t}\right)\right|^{p} d t d r \\
& \geq C \int_{r_{0}}^{1}(1-r)^{p-1} M_{2}^{p}(r, f) M_{2}^{p}\left(r, g^{\prime}\right) d r \\
& \geq C \int_{r_{0}}^{1}(1-r)^{p-1}\left(\log \frac{1}{1-r}\right)^{\frac{p}{2}-1-p \varepsilon} \phi^{p}(r) d r \\
& \geq C \int_{r_{0}}^{1} \frac{d r}{(1-r)\left(\log \frac{1}{1-r}\right)^{p \alpha-\frac{p}{2}+1+p \varepsilon}} \\
& =\infty
\end{aligned}
$$

Since $f \in \mathcal{D}_{p-1}^{p}$, this shows that $\mu_{g, p}$ is not a Carleson measure for $\mathcal{D}_{p-1}^{p}$ and finishes the proof.

\section{Multipliers}

A function $g \in \mathcal{H o l}(\mathbb{D})$ is a multiplier for the space $\mathcal{D}_{\alpha}^{p}$ if $g \mathcal{D}_{\alpha}^{p} \subset \mathcal{D}_{\alpha}^{p}$, that is, if $f g \in \mathcal{D}_{\alpha}^{p}$, for all $f \in \mathcal{D}_{\alpha}^{p}$. By the closed-graph theorem, $g$ is a multiplier for $\mathcal{D}_{\alpha}^{p}$ if and only if there exists a constant $C>0$ such that

$$
\|f g\|_{\mathcal{D}_{\alpha}^{p}} \leq C\|f\|_{\mathcal{D}_{\alpha}^{p}}, \quad \text { for all } f \in \mathcal{D}_{\alpha}^{p} .
$$

The space of all multipliers of the space $\mathcal{D}_{\alpha}^{p}$ will be denoted by $m\left(\mathcal{D}_{\alpha}^{p}\right)$. Since $\mathcal{D}_{\alpha}^{p}$ contains the constant functions, we have $m\left(\mathcal{D}_{\alpha}^{p}\right) \subset \mathcal{D}_{\alpha}^{p}$. Wu obtained in Theorem 4.2 of [23] a characterization of the multipliers of the spaces $\mathcal{D}_{\alpha}^{p}(\alpha>1,0<$ $p<\infty)$. In particular, he proved the following result.

Theorem C. Suppose that $0<p<\infty$ and $g$ is an analytic function in $\mathbb{D}$. Then $g \in$ $m\left(\mathcal{D}_{p-1}^{p}\right)$ if and only if $g \in H^{\infty}$ and the measure $\mu_{g, p}$ on $\mathbb{D}$ defined by $d \mu_{g, p}(z)=$ $(1-|z|)^{p-1}\left|g^{\prime}(z)\right|^{p} d A(z)$ is a Carleson measure for $\mathcal{D}_{p-1}^{p}$.

Theorem C and Theorem B yield the following theorem (see also Theorem 2.2 of $[22])$. 
Theorem D. Suppose that $0<p<2$ and $g$ is an analytic function in $\mathbb{D}$. Then $g \in$ $m\left(\mathcal{D}_{p-1}^{p}\right)$ if and only if $g \in H^{\infty}$ and the measure $\mu_{g, p}$ on $\mathbb{D}$ defined by $d \mu_{g, p}(z)=$ $(1-|z|)^{p-1}\left|g^{\prime}(z)\right|^{p} d A(z)$ is a classical Carleson measure.

Since $\mathcal{D}_{1}^{2}=H^{2}$, we have $m\left(\mathcal{D}_{1}^{2}\right)=m\left(H^{2}\right)=H^{\infty}$. We remark also that even though there is no relation of inclusion beteween $\mathcal{D}_{p-1}^{p}$ and $\mathcal{D}_{q-1}^{q}(p \neq q)$, it is easy to see that

$$
m\left(\mathcal{D}_{p-1}^{p}\right) \subset m\left(\mathcal{D}_{q-1}^{q}\right), \quad \text { if } 0<p \leq q \leq 2 .
$$

Indeed, if $0<p \leq q \leq 2$ and $g \in m\left(\mathcal{D}_{p-1}^{p}\right)$, then $g \in H^{\infty}$ and then it follows that $\sup _{z \in \mathbb{D}}(1-|z|)\left|g^{\prime}(z)\right|=A<\infty$. Then, for every interval $I \subset \mathbb{T}$, we have

$$
\int_{S(I)}(1-|z|)^{q-1}\left|g^{\prime}(z)\right|^{q} d A(z) \leq A^{q-p} \int_{S(I)}(1-|z|)^{p-1}\left|g^{\prime}(z)\right|^{p} d A(z) .
$$

Since $\mu_{g, p}$ is a classical Carleson measure, it follows that $\mu_{g, q}$ is also a classical Carleson measure. This and the fact that $g \in H^{\infty}$ yield that $g \in m\left(\mathcal{D}_{q-1}^{q}\right)$.

Using Theorem D and our results of Section 3 we can obtain sufficient conditions for multipliers of the spaces $\mathcal{D}_{p-1}^{p}, 0<p<2$.

Theorem 5.1. Suppose that $0<q<2$ and let $g$ be an analytic function in $\mathbb{D}$, $g(z)=\sum_{n=0}^{\infty} a_{n} z^{n}(z \in \mathbb{D})$, satisfying

$$
\sum_{n=0}^{\infty}\left(\sum_{k \in I(n)}\left|a_{k}\right|\right)^{q}<\infty
$$

(i) If $0<q \leq 1$ and $q \leq p<2$, then $g \in m\left(\mathcal{D}_{p-1}^{p}\right)$.

(ii) If $0<q \leq p<2$ and $g \in H^{\infty}$, then $g \in m\left(\mathcal{D}_{p-1}^{p}\right)$.

Proof. Notice that if $0<q \leq p$ then (5.2) implies that

$$
\sum_{n=0}^{\infty}\left(\sum_{k \in I(n)}\left|a_{k}\right|\right)^{p}<\infty
$$

Then, using Theorem 3.1 and Theorem D we deduce (ii).

Now, if $0<q \leq 1$ then (5.2) implies $\sum_{k=1}^{\infty}\left|a_{k}\right|<\infty$ and, hence, $g \in H^{\infty}$. Then (i) follows from (ii).

Similarly, using Theorem 3.2, we obtain the following.

Theorem 5.2. Suppose that $0<q \leq 1$ and $q \leq p<2$. Let $g$ be an analytic function in $\mathbb{D}$ which is given by a power series with Hadamard gaps,

$$
g(z)=\sum_{k=1}^{\infty} a_{k} z^{n_{k}} \quad(z \in \mathbb{D}) \quad \text { with } n_{k+1} \geq \lambda n_{k}, \text { for all } k \quad(\lambda>1),
$$

with

Then $g \in m\left(\mathcal{D}_{p-1}^{p}\right)$.

$$
\sum_{k=1}^{\infty}\left|a_{k}\right|^{q}<\infty
$$


We will close the paper studying the connection between the multipliers of the spaces $\mathcal{D}_{q-1}^{q}$ and the spaces $Q_{p}$.

When $0<p<\infty$, an analytic function $f$ in $\mathbb{D}$ belongs to the space $Q_{p}$ if

$$
\sup _{a \in \mathbb{D}} \int_{\mathbb{D}}\left|f^{\prime}(z)\right|^{2} g(z, a)^{p} d A(z)<\infty,
$$

where $g$ denotes the Green function for the disc given by

$$
g(z, a)=\log \left|\frac{1-\bar{a} z}{a-z}\right|, \quad z, a \in \mathbb{D}, \quad z \neq a .
$$

The spaces $Q_{p}$ are conformally invariant. They have their origin in the papers [24] where it was shown that $Q_{2}=\mathcal{B}$ (the Bloch space) and [2] where this result was extended by showing that $Q_{p}=\mathcal{B}$ for all $p>1$. The space $Q_{1}$ coincides with $B M O A$. When $0<p<1, Q_{p}$ is a proper subspace of $B M O A$ and has many interesting properties (see, [8], [3], or the recent detailed monograph [25]).

There are various characterizations of $Q_{p}$ spaces. The one that will be useful for us is expressed in terms of $p$-Carleson measures. Given a positive Borel measure $\mu$ on $\mathbb{D}$, we say that $\mu$ is a $p$-Carleson measure if there exists a positive constant $C$ such that

$$
\mu(S(I)) \leq C|I|^{p}, \quad \text { for every interval } I \subset \mathbb{T} .
$$

The special case $p=1$ yields the classical Carleson measures. The following characterization of $Q_{p}$ spaces was obtained by Aulaskari, Stegenga and Xiao [3].

Theorem E. Let $0<p<\infty$. A function $f$ holomorphic in $\mathbb{D}$ is a member of $Q_{p}$ if and only if the measure $\mu$ on $\mathbb{D}$ defined by $d \mu(z)=\left(1-|z|^{2}\right)^{p}\left|f^{\prime}(z)\right|^{2} d A(z)$ is a p-Carleson measure.

Vinogradov [22] proved that, for $0<s<2$, there are Blaschke products which do not belong to the space $\mathcal{D}_{s-1}^{s}$. Hence,

$$
H^{\infty} \not \subset \mathcal{D}_{s-1}^{s}, \quad \text { and } \quad Q_{1} \not \subset \mathcal{D}_{s-1}^{s}, \quad 0<s<2 .
$$

However, we can prove the following result.

Theorem 5.3. (i) $\bigcup_{0<p<1} Q_{p} \subsetneq \bigcap_{0<s \leq 2} \mathcal{D}_{s-1}^{s}$.

(ii) If $0<p<1$ and $0<s<2$ then $H^{\infty} \cap Q_{p} \subsetneq m\left(\mathcal{D}_{s-1}^{s}\right)$.

Proof of (i). Take $p$ and $s$ with $0<p<1$ and $0<s<2$, and $f \in Q_{p}$. We have

$$
\begin{aligned}
& \int_{\mathbb{D}}\left|f^{\prime}(z)\right|^{s}\left(1-|z|^{2}\right)^{s-1} d A(z) \\
= & \int_{\mathbb{D}}\left[\left|f^{\prime}(z)\right|^{s}\left(1-|z|^{2}\right)^{\frac{s p}{2}}\right]\left[1-|z|^{2}\right]^{\frac{s(2-p)-2}{2}} d A(z) .
\end{aligned}
$$


Applying Hölder's inequality with the exponents $2 / s$ and $\frac{2}{2-s}$, we obtain

$$
\begin{aligned}
& \int_{\mathbb{D}}\left|f^{\prime}(z)\right|^{s}\left(1-|z|^{2}\right)^{s-1} d A(z) \\
& \leq\left[\int_{\mathbb{D}}\left|f^{\prime}(z)\right|^{2}\left(1-|z|^{2}\right)^{p} d A(z)\right]^{s / 2}\left[\int_{\mathbb{D}}\left(1-|z|^{2}\right)^{\frac{s(2-p)-2}{2-s}} d A(z)\right]^{\frac{2-s}{2}} .
\end{aligned}
$$

Theorem E implies that $\int_{\mathbb{D}}\left|f^{\prime}(z)\right|^{2}\left(1-|z|^{2}\right)^{p} d A(z)<\infty$. Also, $\frac{s(2-p)-2}{2-s}>-1$ and then it follows that $\int_{\mathbb{D}}\left(1-|z|^{2}\right)^{\frac{s(2-p)-2}{2-s}} d A(z)<\infty$. Consequently, we see that $\int_{\mathbb{D}}\left|f^{\prime}(z)\right|^{s}\left(1-|z|^{2}\right)^{s-1} d A(z)<\infty$, that is, $f \in \mathcal{D}_{s-1}^{s}$. Thus we have proved that $\bigcup_{0<p<1} Q_{p} \subset \bigcap_{0<s \leq 2} \mathcal{D}_{s-1}^{s}$. To see that the inclusion is strict, let $S$ be the atomic singular inner function defined by

$$
S(z)=\exp \left(\frac{z+1}{z-1}\right), \quad z \in \mathbb{D} .
$$

Then Theorem 2.7 of [22] implies that $S \in \bigcap_{0<s \leq 2} m\left(\mathcal{D}_{s-1}^{s}\right) \subset \bigcap_{0<s \leq 2} \mathcal{D}_{s-1}^{s}$ but Theorem 2.2 of [8] shows that $S \notin \bigcup_{0<p<1} Q_{p}$.

The following simple lemma will be used to prove (ii).

Lemma 5.4. If $\alpha>-1$ then there exists a constant $C>0$ (which depends only on a) such that

$$
\int_{S(I)}\left(1-|z|^{2}\right)^{\alpha} d A(z) \leq C|I|^{\alpha+2},
$$

for all intervals $I \subset \mathbb{T}$.

The proof of the lemma is elementary and will be omitted.

Proof of (ii). Suppose that $0<p<1$ and $0<s<2$ and take $f \in H^{\infty} \cap Q_{p}$. Let $I \subset \mathbb{T}$ be an interval. Applying Hölder's inequality with the exponents $2 / s$ and $\frac{2}{2-s}$, we obtain

$$
\begin{aligned}
& \int_{S(I)}\left|f^{\prime}(z)\right|^{s}\left(1-|z|^{2}\right)^{s-1} d A(z) \\
& \leq\left[\int_{S(I)}\left|f^{\prime}(z)\right|^{2}\left(1-|z|^{2}\right)^{p} d A(z)\right]^{s / 2}\left[\int_{S(I)}\left(1-|z|^{2}\right)^{\frac{s(2-p)-2}{2-s}} d A(z)\right]^{\frac{2-s}{2}} .
\end{aligned}
$$

Since $f \in Q_{p}$, there is a constant $C_{1}$ such that

$$
\int_{S(I)}\left|f^{\prime}(z)\right|^{2}\left(1-|z|^{2}\right)^{p} d A(z) \leq C_{1}|I|^{p} .
$$

On the other hand, since $\frac{s(2-p)-2}{2-s}>-1$, Lemma 5.4 implies that

$$
\int_{S(I)}\left(1-|z|^{2}\right)^{\frac{s(2-p)-2}{2-s}} d A(z) \leq C|I|^{\frac{s(2-p)-2}{2-s}+2},
$$


which, together with (5.6) and (5.7), gives that there is a constant $C_{2}$ such that

$$
\int_{S(I)}\left|f^{\prime}(z)\right|^{s}\left(1-|z|^{2}\right)^{s-1} d A(z) \leq C_{2}|I| .
$$

Thus, the measure $\mu_{f, s}$ is a classical Carleson measure. Since $f \in H^{\infty}$, using Theorem D we deduce that $f \in m\left(\mathcal{D}_{s-1}^{s}\right)$. Hence, we have proved that $Q_{p} \cap H^{\infty} \subset$ $m\left(\mathcal{D}_{s-1}^{s}\right)$. As noticed above, if $S$ is the atomic singular inner function defined by (5.5) then $S$ belongs to $m\left(\mathcal{D}_{s-1}^{s}\right)$ but not to $H^{\infty} \cap Q_{p}$. Hence, the inclusion is strict.

\section{References}

[1] N. Arcozzi, R. Rochberg and E. Sawyer, Carleson measures for analytic Besov spaces, Rev. Mat. Iberoamericana 18 (2002), no. 2, 443-510.

[2] R. Aulaskari and P. Lappan, Criteria for an analytic function to be Bloch and a harmonic or meromorphic function to be normal, In: Complex Analysis and its Applications (Hong Kong, 1993), pp. 136-146, Pitman Res. Notes Math. Ser., 305, Longman Scientific and Technical, Harlow 1994.

[3] R. Aulaskari, D. A. Stegenga, and J. Xiao, Some subclasses of BMOA and their characterizations in terms of Carleson measures, Rocky Mountain J. Math. 26 (1996), no. 2, 485-506.

[4] S. M. Buckley, P. Koskela and D. Vukotić, Fractional integration, differentiation, and weighted Bergman spaces, Math. Proc. Cambridge Philos. Soc. 126 (1999), 369-385.

[5] L. Carleson, Interpolation by bounded analytic functions and the corona problem, Ann. of Math.76 (1962), 547-559.

[6] P. L. Duren, Theory of $H^{p}$ Spaces (Academic Press, New York-London 1970. Reprint: Dover, Mineola, New York 2000).

[7] P. L. Duren and A. P. Schuster, Bergman Spaces, Mathematical Surveys and Monographs, 100, American Mathematical Society, Providence, (2004).

[8] M. Essén and J. Xiao, Some results on $Q_{p}$ spaces, $0<p<1$, J. Reine Angew. Math. 485 (1997), 173-195.

[9] T. M. Flett, The dual of an inequality of Hardy and Littlewood and some related inequalities, J. Math. Aanl. Appl. 38 (1972), 746-765.

[10] J. B. Garnett, Bounded Analytic Functions (Academic Press, New York, etc. 1981).

[11] D. Girela, Mean growth of the derivative of certain classes of analytic functions, Math. Proc. Camb. Phil. Soc. 112 (1992), 335-342.

[12] D. Girela and J. A. Peláez, Growth properties and sequences of zeros of analytic functions in spaces of Dirichlet type, to appear in J. Austral. Math. Soc.

[13] D. Girela and J. A. Peláez, Integral means of analytic functions, Ann. Acad. Sci. Fenn. Math. 29 (2004), 459-469.

[14] W. W. Hastings, A Carleson measure theorem for Bergman spaces, Proc. Amer. Math. Soc. 52 (1975), 237-241.

[15] H. Hedenmalm, B. Korenblum and K. Zhu, Theory of Bergman Spaces, Graduate Texts in Mathematics 199, Springer, New York, Berlin, etc. (2000). 
[16] D. H. Luecking, Inequalities on Bergman spaces, Illinois J. Math. 25 (1981), 1-11.

[17] D. H. Luecking, A technique for characterizing Carleson measures on Bergman spaces, Proc. Amer. Math. Soc., 87 (1983), 656-660.

[18] M. Mateljevic and M. Pavlovic, $L^{p}$ - behaviour of power series with positive coeffcients and Hardy spaces, Proc. Amer. Math. Soc., 87 (1983), 309-316.

[19] V. L. Oleinik, Embedding theorems for weighted classes of harmonic and analytic functions, (in russian), Zap. Nauch. Sem. LOMI Steklov 47 (1974), 120-137; english translation in J. Soviet Math. 9 (1978), 228-243.

[20] V. L. Oleinik and B. S. Pavlov, Embedding theorems for weighted classes of harmonic and analytic functions, (in russian), Zap. Nauch. Sem. LOMI Steklov 22 (1971), 94102; english translation in J. Soviet Math. 2 (1974), 135-142.

[21] D. Stegenga, Multipliers of the Dirichlet spaces, Illinois J. Math. 24 (1980), 113-139.

[22] S. A. Vinogradov, Multiplication and division in the space of analytic functions with area integrable derivative, and in some related spaces, (in russian), Zap. Nauchn. Sem. S.-Peterburg. Otdel. Mat. Inst. Steklov. (POMI) 222 (1995), Issled. po Linein. Oper. i Teor. Funktsii 23, 45-77, 308; english translation in J. Math. Sci. (New York) 87, no. 5 (1997), 3806-3827.

[23] Z. Wu, Carleson measures and multipliers for Dirichlet spaces, J. Funct. Anal. 169 (1999), 148-163.

[24] J. Xiao, Carleson measure, atomic decomposition and free interpolation from Bloch space, Ann. Acad. Sci. Fenn. Ser. A I Math. 19 (1994), no. 1, 35-46.

[25] J. Xiao, Holomorphic Q classes, Lecture Notes in Mathematics 1767, SpringerVerlag, Berlin (2001).

[26] A. Zygmund, Trigonometric Series, Vol. I and II, second edition, Cambridge Univ. Press, Cambridge (1959).

Daniel Girela

Departamento de Análisis Matemático

Facultad de Ciencias

Universidad de Málaga

29071 Málaga

Spain

e-mail: girela@uma.es

José Ángel Peláez

Departamento de Análisis Matemático

Facultad de Ciencias

Universidad de Málaga

29071 Málaga

Spain

e-mail: pelaez@anamat.cie.uma.es 